\title{
Spin relaxation of two-dimensional electrons with a hierarchy of spin-orbit couplings
}

\author{
Yuan Li and You-Quan Li \\ Zhejiang Institute of Modern Physics and Department of Physics, \\ Zhejiang University, Hangzhou 310027, P. R. China
}

(Received December 12, 2018)

\begin{abstract}
The density matrix formalism is applied to calculate the spin-relaxation time for two-dimensional systems with a hierarchy of spin-orbit couplings, such as Rashba-type, Dresselhaus-type and so on. It is found that the spin-relaxation time can be infinite if those coupling strengths $\alpha, \beta, \gamma_{1}$ and $\gamma_{2}$ satisfy either condition (i) $\alpha=\beta, \gamma_{1}=0$ or (ii) $\alpha=-\beta, \gamma_{2}=0$, which correspond to the vanishing Yang-Mills magnetic field. The effect caused by the application of an external magnetic field is also discussed. It is found that the longitudinal and in-plane spin components can possess infinite life time when the spin components, the Larmor precession frequency and the external magnetic field satisfy certain relations.

PACS numbers: $72.25 . \mathrm{Rb}, 72.25 . \mathrm{Dc}$
\end{abstract}

\section{INTRODUCTION}

Spintronics 1], or spin based electronics 2], has absorbed much more attention during the last decade. One important issue in this research area is the manipulation of spin polarized electrons with the help of an electric field [3, 4, 5, 6, 7]. The system with spin-orbit couplings makes these efforts possible and thus brings great interests from both academic and practical aspects 3, 4, 5, 6. 6 . However, the problem of the loss of the average microscopic spin is crucial in experimental data analysis and applicable device construction. The study of the spinrelaxation mechanisms of two-dimensional electrons is thus very important.

Spin relaxation exhibits some properties of the spin dynamics, which plays inevitable role in realizing the applicable spintronics devices. The main mechanism of spin relaxation in systems lacking inversion symmetry is the D'yakonov-Perel's mechanism [8, [9], in which the spin of the electron precesses due to an effective $\mathbf{k}$-dependent magnetic field. For electrons in two-dimensional semiconductor heterostructures or quantum wells, the structure inversion asymmetry brings about the Rashba spin-orbit coupling 10, 11, 12], while the bulk inversion asymmetry in the $A_{3} B_{5}$ compounds leads to the Dresselhaus 13 . spin-orbit coupling. Spin-relaxation time in some semiconductors with both Rashba and Dresselhaus couplings was calculated by analyzing the condition of spin decay 14, 15, and the effect of external magnetic fields was discussed 16 furthermore. An infinite spin-relaxation time [17] was predicted in the system with equal Rashba and Dresselhaus coupling constants by making use of an $\mathrm{SU}(2)$ symmetry in $k$-space. It is important to understand the spin-relaxation mechanism and the condition for infinite spin-relaxation time to occur, which would be helpful for overcoming the difficulties in the spin-based information processes.

In this paper, we develop the aforementioned theory of spin relaxation to describe two-dimensional electron systems in the presence of $U(1)$ Maxwell field and $S U(2)$ Yang-Mills fields. Such a system can be realized in cer- tain semiconductor materials where the spin-orbit couplings, such as Rashba-type, Dresselhaus-type and etc., play crucial roles. Using density matrix formalism, we calculate the spin-relaxation time for the system with a hierarchy of spin-orbit couplings. In the absence of the Maxwell magnetic field, the infinity of the spin-relaxation time occurs if the spin-orbit couplings $\alpha, \beta, \gamma_{1}$ and $\gamma_{2}$ satisfy the condition in which the Yang-Mills magnetic field vanishes. In order to capture the physical essence of the emergence of an infinite spin-relaxation time, we further study the effect of the external magnetic field on the same systems and find that the longitudinal and in-plane spin components can also possess infinite life times when the spin orientation, the Larmor precession frequency and external magnetic field satisfy some relations. Base on the analysis of spin-orbit systems with or without Maxwell magnetic fields, we expose a physics picture for a clear understanding of the infinite spin-relaxation time, which is helpful for the design of the spin-based devices.

\section{SPIN RELAXATION ARISING FROM SPIN-ORBIT COUPLINGS}

To start with a general formalism, we consider the Schrödinger equation for a particle moving in an external $U(1)$ Maxwell field and an $S U(2)$ Yang-Mills field [18],

$$
\begin{aligned}
& i \hbar \frac{\partial}{\partial t} \Psi(r, t)=H \Psi(r, t), \\
& H=\frac{1}{2 m}\left(\hat{\mathbf{p}}-\frac{e}{c} \mathbf{A}-\eta \mathcal{A}^{a} \hat{\tau}^{a}\right)^{2}+e A_{0}+\eta \mathcal{A}_{0}^{a} \hat{\tau}^{a},
\end{aligned}
$$

where $\Psi$ is a two-component wavefunction, $\mathbf{A}_{\mu}=$ $\left(A_{0}, A_{i}\right)$ denotes the vector potential of the Maxwell electromagnetic field, and $\mathbb{A}_{\mu}=\mathcal{A}_{\mu}^{a} \hat{\tau}^{a}$ that of Yang-Mills field with $\hat{\tau}^{a}$ being the generators of $S U(2)$ Lie group. It has been shown [18] that the Yang-Mills fields can be realized in certain semiconductor materials.

Firstly, we consider a two-dimensional system (in $x$ $y$ plane) with a four-parameter Yang-Mills potentials: $\overrightarrow{\mathcal{A}_{0}}=(0,0,0), \overrightarrow{\mathcal{A}_{x}}=\frac{2 m}{\eta \hbar}\left(0, \beta+\alpha, \gamma_{2}\right), \overrightarrow{\mathcal{A}_{y}}=\frac{2 m}{\eta \hbar}(\beta-$ 
$\left.\alpha, 0, \gamma_{1}\right), \overrightarrow{\mathcal{A}}_{z}=(0,0,0)$ where $\alpha, \beta$ and $\gamma$ characterize the strengths of spin-orbit couplings of Rashba-type, Dresselhaus-type, etc., respectively. If writing out the Hamiltonian explicitly, we have

$$
\begin{aligned}
H= & \frac{\hbar^{2} k^{2}}{2 m}+V+k_{y} \sigma_{x}(\alpha-\beta)-k_{x} \sigma_{y}(\alpha+\beta) \\
& -\left(\gamma_{1} k_{y}+\gamma_{2} k_{x}\right) \sigma_{z} \\
= & \frac{\hbar^{2} k^{2}}{2 m}+V+\frac{\hbar}{2} \vec{\sigma} \cdot \Omega_{\mathbf{k}}
\end{aligned}
$$

with $V=2 m\left(\gamma_{1}^{2}+2\left(\alpha^{2}+\beta^{2}\right)+\gamma_{2}^{2}\right) / \hbar^{2}+V_{s c}, \boldsymbol{\Omega}_{\mathbf{k}}=$ $2\left(k_{y}(\alpha-\beta),-k_{x}(\alpha+\beta),-\left(\gamma_{1} k_{y}+\gamma_{2} k_{x}\right)\right) / \hbar$ and $\vec{\sigma}$ being the pauli matrix. Here $m$ stands for the effective mass of electrons in the material, $V_{\mathrm{sc}}=e A_{0}$ the scattering potential which is independent of spin indices. The scattering is supposed to be elastic. The last term in above equation $H^{\prime}=\frac{\hbar}{2} \vec{\sigma} \cdot \boldsymbol{\Omega}_{\mathbf{k}}$ causes the precession of electron spins with the Larmor frequency $\boldsymbol{\Omega}_{\mathbf{k}}$ which can be regarded as an effective magnetic field.

To calculate the spin-relaxation time, we use density matrix formalism. The electron density matrix $\rho(\mathbf{k})$ with components $\rho_{s s^{\prime}}(\mathbf{k}), s, s^{\prime}$ being the indices of electron spin states, is defined by [14, 19]

$$
\frac{\rho(\mathbf{k})}{\tau}+\frac{i}{\hbar}\left[H^{\prime}(\mathbf{k}), \rho(\mathbf{k})\right]+\sum_{k^{\prime}} W_{\mathbf{k k}^{\prime}}\left(\rho(\mathbf{k})-\rho\left(\mathbf{k}^{\prime}\right)\right)=0
$$

where $\tau$ is the lifetime, $W_{\mathbf{k k}^{\prime}}$ is the scattering probability from $\mathbf{k}$ to $\mathbf{k}^{\prime}$ and the square bracket denotes commutator.

Since $H^{\prime}$ contributes merely a small perturbation, the spin-relaxation time is much longer than the time for electron-momentum distribution to become isotropic, i.e., $\tau \gg \tau_{1}, \tau_{1}$ the momentum relaxation time. Therefore, it is convenient to split the density matrix into two parts,

$$
\rho=\bar{\rho}+\rho^{\prime}, \quad \text { with } \overline{\rho^{\prime}}=0
$$

Here we use a bar to denote an average taken over all directions of $\mathbf{k}$ and a prime to denote the deviation ones, $\rho^{\prime}(\mathbf{k}) \ll \bar{\rho}$. Taking average for Eq. (3), we have the following relation,

$$
\frac{\bar{\rho}}{\tau}+\frac{i}{\hbar} \overline{\left[H^{\prime}(\mathbf{k}), \rho^{\prime}(\mathbf{k})\right]}=0 .
$$

Eq. (3) can also be written out as

$$
\begin{aligned}
\frac{\rho^{\prime}(\mathbf{k})}{\tau}+ & \frac{i}{\hbar}\left[H^{\prime}(\mathbf{k}), \rho^{\prime}(\mathbf{k})\right]-\frac{i}{\hbar} \overline{\left[H^{\prime}(\mathbf{k}), \rho^{\prime}(\mathbf{k})\right]}+\frac{i}{\hbar}\left[H^{\prime}(\mathbf{k}), \bar{\rho}\right] \\
& +\sum_{\mathbf{k}^{\prime}} W_{\mathbf{k k}^{\prime}}\left[\rho^{\prime}(\mathbf{k})-\rho^{\prime}\left(\mathbf{k}^{\prime}\right)\right]=0
\end{aligned}
$$

in which Eq. (5) has been used. Without taking account of the higher order terms, we need to solve the following equation

$$
\frac{i}{\hbar}\left[H^{\prime}(\mathbf{k}), \bar{\rho}\right]+\sum_{\mathbf{k}^{\prime}} W_{\mathbf{k} \mathbf{k}^{\prime}}\left[\rho^{\prime}(\mathbf{k})-\rho^{\prime}\left(\mathbf{k}^{\prime}\right)\right]=0 .
$$

This approximation is valid when $\Omega_{k} \tau_{1} \ll 1$. For elastic process the scattering probability is a function of deflection angle only, which makes it possible to expand the above equation in terms of Fourier series. After some algebra, one can express $\rho^{\prime}$ in terms of $\bar{\rho}$. Substituting it into Eq. (5) and employing the Boltzmann equation with only collision term, we can obtain the rate of averaged density matrix $\bar{\rho}$, namely,

$$
\left(\frac{\partial \bar{\rho}}{\partial t}\right)_{\text {sp.rel. }}=-\frac{1}{\hbar^{2}} \sum_{n} \tau_{n}\left[H_{-n}^{\prime},\left[H_{n}^{\prime}, \bar{\rho}\right]\right]
$$

with

$$
\begin{aligned}
H_{n}^{\prime} & =\oint \frac{d \phi_{\mathbf{k}}}{2 \pi} H^{\prime}(\mathbf{k}) \exp \left(-i n \phi_{\mathbf{k}}\right), \\
\frac{1}{\tau_{n}} & =\oint d \theta W_{\mathbf{k k}^{\prime}}(1-\cos n \theta),
\end{aligned}
$$

where $\phi_{\mathbf{k}}$ is the angle between $\mathbf{k}$ and $x$-axis, and $\theta=$ $\phi_{\mathbf{k}}-\phi_{\mathbf{k}^{\prime}}$.

Now we are in the position to investigate the kinetics of the spin density $S_{i}(t)=\int a_{i}(\varepsilon, t) d \varepsilon$, where $a_{i}(\varepsilon, t)=$ $\left[F_{+}(\varepsilon)-F_{-}(\varepsilon)\right] s_{i}(t), s_{i}(t)=\operatorname{tr}\left(\sigma_{\mathrm{i}} \bar{\rho}\right)$ and $F_{ \pm}(\varepsilon)$ refers to the distribution function projected along the direction parallel or anti-parallel to $\mathbf{s}=\left(s_{1}, s_{2}, s_{3}\right)$. Accordingly, we obtain the evolution equation for the spin density at the time longer than $\tau_{1}$ [15]:

$$
\begin{aligned}
& \dot{S}_{i}(t)=-\frac{1}{\tau_{i j}} S_{j}(t) \\
& \frac{1}{\tau_{i j}}=\frac{1}{2 \hbar^{2}} \sum_{-\infty}^{\infty} \frac{\int d \varepsilon\left(F_{+}-F_{-}\right) \tau_{n} \operatorname{tr}\left\{\left[\mathrm{H}_{-\mathrm{n}}^{\prime},\left[\mathrm{H}_{\mathrm{n}}^{\prime}, \bar{\rho}\right]\right] \sigma_{\mathrm{i}}\right\}}{\int d \varepsilon\left(F_{+}-F_{-}\right)},
\end{aligned}
$$

where $i, j=x, y, z$.

For the Hamiltonian under consideration (2), we obtain the following,

$$
\begin{aligned}
& \frac{1}{\tau_{x x}}=\frac{\gamma_{1}^{2}+\gamma_{2}^{2}+(\alpha+\beta)^{2}}{2} \Lambda, \\
& \frac{1}{\tau_{y y}}=\frac{\gamma_{1}^{2}+\gamma_{2}^{2}+(\alpha-\beta)^{2}}{2} \Lambda, \\
& \frac{1}{\tau_{z z}}=\left(\alpha^{2}+\beta^{2}\right) \Lambda, \\
& \frac{1}{\tau_{x z}}=\frac{1}{\tau_{z x}}=\frac{\gamma_{1}(\alpha-\beta)}{2} \Lambda, \\
& \frac{1}{\tau_{y z}}=\frac{1}{\tau_{z y}}=-\frac{\gamma_{2}(\alpha+\beta)}{2} \Lambda, \\
& \frac{1}{\tau_{x y}}=\frac{1}{\tau_{y x}}=0,
\end{aligned}
$$

where the coefficient $\Lambda$ is given by

$$
\Lambda=\frac{8 m}{\hbar^{4}} \frac{\int d \varepsilon\left[F_{+}(\varepsilon)-F_{-}(\varepsilon)\right] \tau_{1}(\varepsilon) \varepsilon}{\int d \varepsilon\left[F_{+}(\varepsilon)-F_{-}(\varepsilon)\right]} .
$$


The above entities in Eq. (12) define the spin-relaxation tensor $\Gamma=\operatorname{mat}\left(\frac{1}{\tau_{i j}}\right)$. Diagonalizing this matrix (see Appendix $\AA$ for details), we obtain

$$
\mathbb{T}^{-1}=\frac{\Lambda}{2}\left(\begin{array}{ccc}
\frac{1}{\tau_{\perp}} & 0 & 0 \\
0 & \frac{1}{\tau_{\|, \pm}} & 0 \\
0 & 0 & \frac{1}{\tau_{\|, \pm}}
\end{array}\right)
$$

with

$$
\begin{aligned}
\frac{1}{\tau_{\|, \pm}} & =\frac{1}{2}\left\{\frac{1}{\tau_{\perp}} \pm \sqrt{\left(\gamma_{1}^{2}+\gamma_{2}^{2}\right)^{2}+8 \alpha \beta\left(\gamma_{2}^{2}-\gamma_{1}^{2}+2 \alpha \beta\right)}\right\} \\
\frac{1}{\tau_{\perp}} & =\gamma_{1}^{2}+\gamma_{2}^{2}+2\left(\alpha^{2}+\beta^{2}\right)
\end{aligned}
$$

Clearly, two of the diagonal elements are always positively definite and the other one $1 / \tau_{\|,-}$is not. The condition for a vanishing $1 / \tau_{\|,-}$turns out to be

$$
\gamma_{1}^{2}(\alpha+\beta)^{2}+\gamma_{2}^{2}(\alpha-\beta)^{2}+(\alpha+\beta)^{2}(\alpha-\beta)^{2}=0 .
$$

The above equation gives rise to two solutions

$$
\begin{aligned}
& \text { (i) } \alpha=\beta, \quad \gamma_{1}=0, \\
& \text { (ii) } \alpha=-\beta, \gamma_{2}=0 .
\end{aligned}
$$

Under these conditions, $\mathbb{T}_{y y}^{-1}$ and $\mathbb{T}_{z z}^{-1}$ are zero when the infinite spin-relaxation times emerge.

Actually, the Yang-Mills "magnetic" field $\mathbb{B}_{z}=b^{a} \hat{\tau}^{a}$ can be calculated, namely

$$
\begin{aligned}
b_{1} & =\frac{4 m^{2}}{\eta^{2} \hbar^{2}}(\beta+\alpha) \gamma_{1}, \\
b_{2} & =\frac{4 m^{2}}{\eta^{2} \hbar^{2}}(\beta-\alpha) \gamma_{2}, \\
b_{3} & =\frac{4 m^{2}}{\eta^{2} \hbar^{2}}\left(\alpha^{2}-\beta^{2}\right) .
\end{aligned}
$$

The condition (15) is also equivalent to

$$
|\vec{b}|^{2}=0, \quad \vec{b}=\left(b_{1}, b_{2}, b_{3}\right),
$$

which implies that the module of the Yang-Mills "magnetic" field vanishes. In other words, the spin-relaxation time can be infinite when the module of the Yang-Mills magnetic field is null. This result is expected to be a criterion to evaluate whether there has an infinite spinrelaxation time in two-dimensional systems with spinorbit couplings. In order to determine which spin component has an infinite life time, the spin precession needs to be analyzed concretely.

The nonvanishing spin orbit coupling $\gamma_{1}$ or $\gamma_{2}$ will bring about some new features which may be useful for possible design with an infinite spin relaxation time. For the first case $\alpha=\beta$ and $\gamma_{1}=0$ in Eq. (16), the Hamiltonian $H^{\prime}$ reduces to

$$
H^{\prime}=\frac{\hbar}{2} \vec{\sigma} \cdot \boldsymbol{\Omega}_{\mathbf{k}}=-k_{x}\left(2 \alpha \sigma_{y}+\gamma_{2} \sigma_{z}\right)
$$

The orientation of Larmor precession frequency $\boldsymbol{\Omega}_{\mathrm{k}}$ is parallel to $y^{\prime}$-axis as illustrated in Fig. 3 in appendix A, thus one can understand why the life time of the $S_{y^{\prime}}$ component is infinite while the other two decay. Here the $y^{\prime}$-axis is defined in the diagonalization procedure of the spin-relaxation time tensor Eq.(12) for the first case given in appendix $\mathrm{A}$

From the appendix $\mathrm{A}$ and the figure therein, we can see that the strengths of spin-orbit coupling $\gamma_{2}$ and $\alpha$ determine the angle $\theta^{\prime}$ between $\boldsymbol{\Omega}_{\mathrm{k}}$ and $z$-axis. The angle $\theta^{\prime}$ can be manipulated by these two parameters, thus a definite alignment of spin with infinite life time can be realized with the help of tuning spin-orbit coupling strengths. On the other hand, the ratio of different type of spin-orbit coupling constants can be determined by means of measuring spin-relaxation time experimentally.

For the second case in Eq. (16), similar analysis can be carried out, which is omitted here. In the special case when both $\gamma_{1}$ and $\gamma_{2}$ vanish, one component of the tensor of spin-relaxation time becomes null, i.e., $1 / \tau_{x x}=0$ or $1 / \tau_{y y}=0$, and thus the $S_{x}$ or $S_{y}$ has infinite life time, which is just the case considered in Ref. [14].

\section{SPIN RELAXATION AFFECTED BY EXTERNAL MAGNETIC FIELD}

In previous section, we considered the case of $\overrightarrow{\mathcal{A}_{0}}=0$ which means external magnetic field is absent. In the presence of the magnetic field, we should take account of

$$
\overrightarrow{\mathcal{A}_{0}}=-\frac{2 \mu_{B}}{\eta}\left(B_{x}, B_{y}, B_{z}\right)
$$

where $\mu_{B}$ is the Bohr magneton. The existence of an external magnetic field is known to affect the dynamics of the electron's spin. The Larmor precession of electron's spin around a sufficiently strong longitudinal magnetic field will suppress the precession about the internal random magnetic fields [20]. The cyclotron motion will change the wave vector $\mathbf{k}$ and affect the spin relaxation due to the DP mechanism. The density-matrix formalism is applicable for calculating electrons' spin-relaxation time. It will be convenient to expand the density matrix for electrons in terms of the unit and Pauli matrices,

$$
\rho(\mathbf{k})=f_{\mathbf{k}}+\mathbf{s}_{\mathbf{k}} \cdot \vec{\sigma}
$$

where $f_{\mathbf{k}}=\operatorname{tr}[\rho(\mathbf{k}) / 2]$ is the spin-averaged-electrondistribution function and $\mathbf{s}_{\mathbf{k}}=\operatorname{tr}[\rho(\mathbf{k}) \vec{\sigma}]$ is the spin per $\mathbf{k}$-state electron. The kinetic equation for the spin distribution is given by [16, 21, 22]:

$$
\begin{aligned}
\frac{\partial \mathbf{s}_{\mathbf{k}}}{\partial t} & +\mathbf{s}_{\mathbf{k}} \times\left(\vec{\omega}_{L}+\boldsymbol{\Omega}_{\mathbf{k}}\right)+\vec{\omega}_{C} \cdot\left[\mathbf{k} \times \nabla_{\mathbf{k}} \mathbf{s}_{\mathbf{k}}\right] \\
& +\sum_{\mathbf{k}^{\prime}} W_{\mathbf{k k}^{\prime}}\left(\mathbf{s}_{\mathbf{k}}-\mathbf{s}_{\mathbf{k}^{\prime}}\right)=0
\end{aligned}
$$

in which the second term refers to spin precession caused by spin-orbit couplings given in Eq. (2) together with 
the external magnetic field; the third term is related to the wave vector variations due to the cyclotron motion whose frequency is $\omega_{C}=\frac{e B_{z}}{m c}$, and the last term denotes the collision integral. Since the internal random magnetic field is regarded as a perturbation, i.e., $\boldsymbol{\Omega}_{\mathbf{k}} \tau_{1} \ll 1$, we can split the spin distribution function $\mathbf{s}_{\mathbf{k}}$ in the following,

$$
\mathbf{s}_{\mathbf{k}}=\mathbf{s}_{k}^{0}+\delta \mathbf{s}_{\mathbf{k}}
$$

where $\mathbf{s}_{k}^{0}$ is a quasi-equilibrium distribution function and thus is independent of direction of $\mathbf{k}$. Whereas, $\delta \mathbf{s}_{\mathbf{k}}$ is a nonequilibrium correction arising from spin-orbit couplings as well as other internal random magnetic fields and thus it contains only first angular harmonics of the spin distribution [23] because elastic scattering processes is taken into account only, accordingly

$$
\delta \mathbf{s}_{\mathbf{k}}=\mathbf{R}_{1} \cos \left(\phi_{k}\right)+\mathbf{R}_{2} \sin \left(\phi_{k}\right),
$$

where the two vectors $\mathbf{R}_{1}$ and $\mathbf{R}_{2}$ are irrelevant to the direction of the wave vector $\mathbf{k}$ though they are functions of the module of $\mathbf{k}$ in general. Substituting Eq. (22) and (23) into Eq. (21), we obtain the following equations:

$$
\begin{aligned}
\frac{d \mathbf{s}_{k}^{0}}{d t} & +\mathbf{s}_{k}^{0} \times \vec{\omega}_{L}+\delta \mathbf{s}_{\mathbf{k}} \times \boldsymbol{\Omega}_{\mathbf{k}}=0 \\
\frac{d \delta \mathbf{s}_{k}}{d t} & +\mathbf{s}_{k}^{0} \times \boldsymbol{\Omega}_{\mathbf{k}}+\delta \mathbf{s}_{\mathbf{k}} \times \vec{\omega}_{L} \\
& +\vec{\omega}_{C} \cdot\left[\mathbf{k} \times \nabla_{\mathbf{k}} \delta \mathbf{s}_{\mathbf{k}}\right]+\frac{\delta \mathbf{s}_{\mathbf{k}}}{\tau_{1}}=0 .
\end{aligned}
$$

where $\tau_{1}$ is momentum-relaxation time whose definition is also given by Eq. (10) for $n=1$. In the light of the number of total electrons $N=2 \sum_{\mathbf{k}} f_{k}$ and the single electron spin $\mathbf{S}^{0}=\frac{\sum_{\mathbf{k}} \mathbf{s}_{\mathbf{k}}}{N}$, summing Eq. (24) over the wave vectors, we obtain the balance equation describing electron's spin relaxation

$$
\frac{d \mathbf{S}^{0}}{d t}+\mathbf{S}^{0} \times \vec{\omega}_{L}+\hat{\Gamma} \mathbf{S}^{0}=0
$$

where the spin-relaxation tensor $\hat{\Gamma}$ referring to the inverse of spin-relaxation times is defined as

$$
\hat{\Gamma} \mathbf{S}^{0}=\frac{1}{N} \sum_{\mathbf{k}} \delta \mathbf{s}_{k} \times \boldsymbol{\Omega}_{\mathbf{k}}
$$

The nonequilibrium correction $\delta \mathbf{s}_{\mathbf{k}}$ can be obtained from Eq. (25) in which the contribution of the rate $d \delta \mathbf{s}_{\mathbf{k}} / d t$ is negligible because its magnitude is of higher order in $\Omega_{\mathbf{k}} \tau_{1}$.

Firstly, we rotate the original coordinate $\{\hat{x}, \hat{y}, \hat{z}\}$ which is related to the principal crystal axes to the new one $\left\{\hat{x}^{\prime}, \hat{y}^{\prime}, \hat{z}^{\prime}\right\}$ (illustrated in Fig. 11). The coordinates in both systems are related, $(\hat{x}, \hat{y}, \hat{z})=\left(\hat{x}^{\prime}, \hat{y}^{\prime}, \hat{z}^{\prime}\right) R^{T}$, by

$$
R=\left(\begin{array}{ccc}
\cos \theta \cos \varphi, & -\sin \varphi, & \sin \theta \cos \varphi \\
\cos \theta \sin \varphi, & \cos \varphi, & \sin \theta \sin \varphi \\
-\sin \theta, & 0, & \cos \theta
\end{array}\right)
$$

Here, $\theta$ is the angle between $z$ and $z^{\prime}$, and $\varphi$ the angle between $y^{\prime}$ and $y([010])$. Similar equations are valid for momentum components, $k_{i}=R_{i j} k_{j}^{\prime}$ (here $i, j=x, y, z$ ). It is convenient to calculate the nonequilibrium correction $\delta \mathbf{s}_{\mathbf{k}}$ and the components of the spin-relaxation tensor in the new frame of coordinate where the Larmor frequency vector $\vec{\omega}_{L}=\frac{\mu_{B}}{\hbar} B_{j} \hat{e}_{j}$ in the original coordinate becomes $\vec{\omega}_{L}=\omega_{L} \hat{z}^{\prime}$ in the new coordinate.

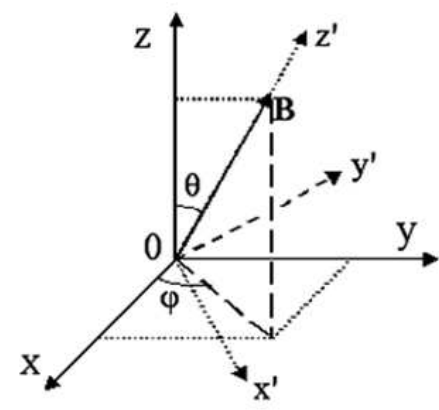

FIG. 1: The scheme of coordinate frames. $z$-axis parallel with the [001] growth axis. $\theta$ and $\varphi$ are the polar and the azimuthal angles of the external magnetic field $\mathbf{B}$. $z^{\prime}$ is chosen in alignment with the orientation of $\mathbf{B}, y^{\prime}$ is lying in $x-y$ plane, and $x^{\prime}$ is chosen to form a right-hand triple with $y^{\prime}$ and $z^{\prime}$.

After tedious calculation, we obtain the spin-relaxation tensor (inverse of the spin-relaxation time) $\hat{\boldsymbol{\Gamma}}$ for degenerate electrons with Fermi energy $E_{F}$ which is given in appendix [B] These results are valid for arbitrary random internal magnetic field and arbitrary orientation of the external field, from which we obtain several conclusions that will be illustrated respectively.

\section{A. Longitudinal relaxation}

The longitudinal spin-relaxation rate is $1 / \tau_{L}=\Gamma_{z^{\prime} z^{\prime}}$. From Eq. (B1), we can obtain the following conclusion: $\Gamma_{z^{\prime} z^{\prime}}=0$ when either

$$
\alpha=\beta, \gamma_{1}=0, \varphi=\pi / 2, \theta=\tan ^{-1}\left(2 \alpha / \gamma_{2}\right),
$$

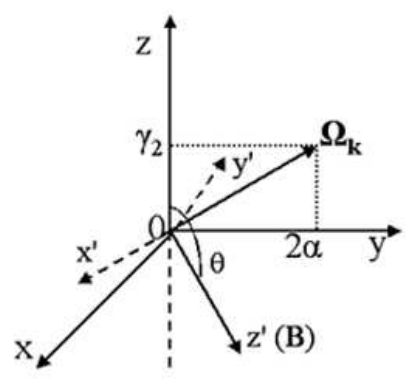

Fig. 2: Schematic of the case $\Gamma_{x^{\prime} x^{\prime}}=0 . \quad \alpha=\beta, \gamma_{1}=0$, $\varphi=\pi / 2, \theta=\tan ^{-1}\left(-\gamma_{2} / 2 \alpha\right)$, the external magnetic field $\mathbf{B}$ is parallel to $z^{\prime}$-direction, and $x^{\prime}$ axis is antiparallel to the Larmor frequency $\boldsymbol{\Omega}_{\mathbf{k}}$. 
or

$$
\alpha=-\beta, \gamma_{2}=0, \varphi=0, \theta=-\tan ^{-1}\left(2 \alpha / \gamma_{1}\right) .
$$

When the longitudinal spin-relaxation time $\tau_{L}$ is infinite, the spin component $S_{z^{\prime}}$ has an infinite life time. Certainly, the Hamiltonian $H^{\prime}$ describing the electron spin precession arising from spin-orbit coupling can also be written as Eq. (18) for the former case in Eq. (29). One can see from Fig. (3) that $S_{z^{\prime}}$ is the component parallel to $\boldsymbol{\Omega}_{\mathbf{k}}$ when $\theta^{\prime}=\theta=\tan ^{-1}\left(2 \alpha / \gamma_{2}\right)$ and $\varphi=\pi / 2$. Thus the infinite life time of $S_{z^{\prime}}$ can easily be understood from physical point of view, that is to say, $S_{z^{\prime}}$ will not precess about Larmor precession frequency $\boldsymbol{\Omega}_{\mathbf{k}}$ and external magnetic $\mathbf{B}$ when $\boldsymbol{\Omega}_{\mathbf{k}}$ is parallel with $\mathbf{B}$. While the other components $S_{x^{\prime}}$ and $S_{y^{\prime}}$ have finite life time due to the precession around $\boldsymbol{\Omega}_{\mathbf{k}}$ arising from internal random magnetic field. If both of the strength of spin-orbit couplings $\gamma_{1}$ and $\gamma_{2}$ vanish, the longitudinal component of the tensor $\Gamma_{z^{\prime} z^{\prime}}$ is zero when $\theta=\pi / 2, \varphi=\pi / 2$, which can be seen from the Eq. (B1). This recovers the special case discussed in Ref. [16].

For the latter case in Eq. (29), the Hamiltonian $H^{\prime}$ becomes

$$
H^{\prime}=\frac{\hbar}{2} \vec{\sigma} \cdot \boldsymbol{\Omega}_{\mathbf{k}}=k_{y}\left(2 \alpha \sigma_{x}-\gamma_{1} \sigma_{z}\right) .
$$

The Larmor frequency $\boldsymbol{\Omega}_{\mathbf{k}}=\frac{2 k_{y}}{\hbar}\left(2 \alpha, 0,-\gamma_{1}\right)$ parallels to the external magnetic field $\mathbf{B}(\theta=$ $\left.-\tan ^{-1}\left(2 \alpha / \gamma_{1}\right), \varphi=0\right)$. So $S_{z^{\prime}}$ does not decay because $\boldsymbol{\Omega}_{\mathrm{k}}$ parallels to $\mathbf{B}$.

\section{B. Transverse relaxation}

Let us analyze the spin relaxation in the plane perpendicular to the external magnetic field. One can find that the transverse components of spin-relaxation tensor can also be zero (i.e., $\Gamma_{x^{\prime} x^{\prime}}=0, \Gamma_{x^{\prime} y^{\prime}}=0, \Gamma_{y^{\prime} x^{\prime}}=0$ ) when either

$$
\alpha=\beta, \gamma_{1}=0, \varphi=\pi / 2, \theta=-\tan ^{-1}\left(\frac{\gamma_{2}}{2 \alpha}\right),
$$

or

$$
\alpha=-\beta, \gamma_{2}=0, \varphi=0, \theta=\tan ^{-1}\left(\frac{\gamma_{1}}{2 \alpha}\right) .
$$

As shown in Fig. 2] the $x^{\prime}$ axis is antiparallel to the Larmor frequency (i.e., $\hat{x}^{\prime} \|-\boldsymbol{\Omega}_{\mathbf{k}}$ ) for the former case in Eq. (31). The spin component $S_{x^{\prime}}$ does not precess about the Larmor precession frequency $\boldsymbol{\Omega}_{\mathbf{k}}$. We know that $\mathbf{B}$ is perpendicular to $\boldsymbol{\Omega}_{\mathrm{k}}$ from Fig. 2 thus $S_{x^{\prime}}$ will precess about the constant external magnetic field $\mathbf{B}$ in the plane paralleling with $\boldsymbol{\Omega}_{\mathbf{k}}$. So the random internal magnetic fields and external magnetic field can not induce spin relaxation for the spin component $S_{x^{\prime}}$. Then $S_{x^{\prime}}$ has an infinite life time associating with $\Gamma_{x^{\prime} x^{\prime}}=0$. And the admixture of the $x^{\prime}$ component to $y^{\prime}$ component of spin-relaxation tensor (which is described by $\Gamma_{x^{\prime} y^{\prime}}$, $\left.\Gamma_{y^{\prime} x^{\prime}}\right)$ are zero, namely $\Gamma_{x^{\prime} y^{\prime}}=0$ and $\Gamma_{y^{\prime} x^{\prime}}=0$ which can also be calculated from Eqs. (B1 B5).

For the latter case in Eq. (31), the direction of the external magnetic field is also perpendicular to the Larmor frequency $\boldsymbol{\Omega}_{\mathbf{k}}$ as illustrated in Eq. (30). The spin component $S_{x^{\prime}}$ is antiparallel to the Larmor frequency $\left(\hat{x}^{\prime} \|-\boldsymbol{\Omega}_{\mathbf{k}}\right)$. The random internal magnetic field and external magnetic field will not induce spin relaxation for the spin component $S_{x^{\prime}}$ due to the same reason as aforementioned. Then $S_{x^{\prime}}$ has an infinite life time, and the components of spin-relaxation tensor $\Gamma_{x^{\prime} y^{\prime}}, \Gamma_{y^{\prime} x^{\prime}}$ vanish (see Eqs. (B1 B5).

The components $\Gamma_{x^{\prime} z^{\prime}}$ and $\Gamma_{y^{\prime} z^{\prime}}$ are smaller than others when the external magnetic field is sufficiently strong $\left(\Omega_{k}^{2} \tau_{1} \ll \omega_{\mathbf{L}}\right)$. Under this condition the in-plane spin components rapidly rotate and the admixture of the in plane components to $z^{\prime}$-component (which is described by $\left.\Gamma_{x^{\prime} z^{\prime}}, \Gamma_{y^{\prime} z^{\prime}}\right)$ plays no role in the spin dynamics. Therefore the above result manifests the general solutions of spin-relaxation time for the Hamiltonian Eq. (2).

\section{SUMMARY}

In the above, we developed a consistent theory of spin dynamics to describe particles moving in an external $U(1)$ Maxwell field and an $S U(2)$ Yang-Mills field which characterizes spin-orbit couplings in certain semiconductors (such as Rashba-type, Dresselhaus-type or other complex types). We used the density-matrix formalism to calculate the spin-relaxation time in such systems in the absence and in the presence of the external magnetic field, respectively. In the absence of external magnetic field, we find that the spin component $S_{y^{\prime}}$ or $S_{z^{\prime}}$ has an infinite life time if the strengths of spin orbit couplings $\alpha, \beta, \gamma_{1}$ and $\gamma_{2}$ satisfy either (i) $\alpha=\beta, \gamma_{1}=0$ or (ii) $\alpha=-\beta, \gamma_{2}=0$. In such a case, the Yang-Mills magnetic field vanishes. From these conditions, the direction of the spin component with infinite life time can be manipulated by tuning $\alpha, \gamma_{2}$ or $\beta, \gamma_{1}$ respectively. In the presence of the external magnetic field, we considered the magnetic effect on the two-dimensional system. We obtained that the longitudinal spin-relaxation time is infinite when the $S_{z^{\prime}}$ is parallel to $\boldsymbol{\Omega}_{\mathbf{k}}$ and $\mathbf{B}$ if either (i') $\alpha=\beta, \gamma_{1}=0, \varphi=\pi / 2, \theta=\tan ^{-1}\left(2 \alpha / \gamma_{2}\right)$ or (ii') $\alpha=-\beta, \gamma_{2}=0, \varphi=0, \theta=-\tan ^{-1}\left(2 \alpha / \gamma_{1}\right)$. By making use of analysis in detail, we conclude that the in-plane spin component $S_{x^{\prime}}$ can also have infinite life time if $S_{x^{\prime}}$ is antiparallel with $\boldsymbol{\Omega}_{\mathbf{k}}$ and perpendicular to $\mathbf{B}$ for either (iii') $\alpha=\beta, \gamma_{1}=0, \varphi=\pi / 2, \theta=\tan ^{-1}\left(-\gamma_{2} / 2 \alpha\right)$ or (iv') $\alpha=-\beta, \gamma_{2}=0, \varphi=0, \theta=\tan ^{-1}\left(\gamma_{1} / 2 \alpha\right)$. That is to say, electron spins have infinite life times if they precess in the plane parallel to the vector of the Larmor precession frequency arising from spin-orbit couplings. These solutions provide a better understanding on the spin dynamics of two-dimensional system with a four-parameter Yang-Mills potentials, which characterizes a hierarchy of 
spin-orbit coupling in certain semiconductor materials. It is expected to expose some more clues for manipulating spin via certain spin-orbit couplings in semiconductors or elaborating spintronics storage devices with long spin-relaxation time.

\section{Acknowledgement}

We acknowledge helpful communications with M. M. Glazov. This work is supported by NSFC No. 10225419 and No. 10674117.

\section{APPENDIX A: ON THE DIAGONALIZING BASES}

We can easily obtain the matrix $U$

$$
U=\left(\begin{array}{ccc}
1 & 0 & 0 \\
0 & \frac{2 \alpha}{\sqrt{\gamma_{2}^{2}+4 \alpha^{2}}} & \frac{-\gamma_{2}}{\sqrt{\gamma_{2}^{2}+4 \alpha^{2}}} \\
0 & \frac{\gamma_{2}}{\sqrt{\gamma_{2}^{2}+4 \alpha^{2}}} & \frac{2 \alpha}{\sqrt{\gamma_{2}^{2}+4 \alpha^{2}}}
\end{array}\right)
$$

which diagonalizes the spin-relaxation tensor given by Eq. (12) to be Eq. (13),

$$
\mathbb{T}^{-1}=U^{-1} \Gamma U
$$

This matrix $U$ turns the evolution equation for the spin density Eq.(11) to be,

$$
\begin{aligned}
\frac{d S^{\prime}}{d t} & =U^{-1} \frac{d S}{d t}=-\left(U^{-1} \Gamma U\right) U^{-1} S \\
& =-\mathbb{T}^{-1} S^{\prime}
\end{aligned}
$$

Hence, the new spin components $S^{\prime}=U^{-1} S$ is obtained

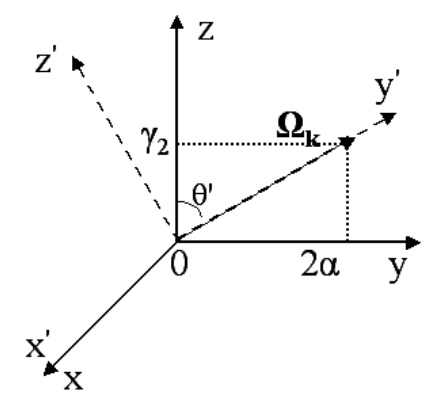

Fig. 3: The orientation of the Larmor frequency $\boldsymbol{\Omega}_{\mathbf{k}}$ for the case $\alpha=\beta, \gamma_{1}=0$, which defines the $y^{\prime}$-axis of the new frame of coordinate

$$
\begin{aligned}
S_{x}^{\prime} & =S_{x} \\
S_{y}^{\prime} & =\sin \theta^{\prime} S_{y}+\cos \theta^{\prime} S_{z}, \\
S_{z}^{\prime} & =-\cos \theta^{\prime} S_{y}+\sin \theta^{\prime} S_{z},
\end{aligned}
$$

where $\tan \theta^{\prime}=2 \alpha / \gamma_{2}$. This means the existence of spinorbit coupling $\gamma_{2}$ makes the orientation of the Larmor frequency $\boldsymbol{\Omega}_{\mathbf{k}}$ to change from $y$ to $y^{\prime}$-axis. As illustrated in Fig. 3] the $\theta^{\prime}$ refers to the angle between the Larmor frequency and $z$-axis.

\section{APPENDIX B: THE SPIN-RELAXATION TENSOR}

In the presence of magnetic field, we obtained the following spin-relaxation tensor

$$
\begin{aligned}
& \Gamma_{z^{\prime} z^{\prime}}=\frac{2 k^{2} \tau_{1}}{\hbar^{2} D_{+} D_{-}}\left\{\left[1+\tau_{1}^{2}\left(\omega_{c}^{2}+\omega_{L}^{2}\right)\right] \times\left[(\alpha+\beta)^{2} \cos ^{2} \varphi+(\alpha-\beta)^{2} \sin ^{2} \varphi\right.\right. \\
& \left.+\left(\gamma_{2} \sin \theta-(\alpha+\beta) \cos \theta \sin \varphi\right)^{2}+\left(\gamma_{1} \sin \theta+(\alpha-\beta) \cos \theta \cos \varphi\right)^{2}\right] \\
& \left.+4 \tau_{1}^{2} \omega_{C} \omega_{L}\left[\left(\alpha^{2}-\beta^{2}\right) \cos \theta+(\alpha+\beta) \gamma_{1} \sin \theta \cos \varphi+(\beta-\alpha) \gamma_{2} \sin \theta \sin \varphi\right]\right\} \\
& \Gamma_{x^{\prime} x^{\prime}}=\frac{2 k^{2} \tau_{1}}{\hbar^{2}}\left\{\frac{1+\left(\omega_{c}^{2}+\omega_{L}^{2}\right) \tau_{1}^{2}}{D_{+} D_{-}} \times\left[\left(\gamma_{2} \cos \theta+(\alpha+\beta) \sin \theta \sin \varphi\right)^{2}\right.\right. \\
& \left.\left.+\left(\gamma_{1} \cos \theta-(\alpha-\beta) \sin \theta \cos \varphi\right)^{2}\right]+\frac{(\alpha-\beta)^{2} \sin ^{2} \varphi+(\alpha+\beta)^{2} \cos ^{2} \varphi}{1+\omega_{C}^{2} \tau_{1}^{2}}\right\} \\
& \Gamma_{y^{\prime} y^{\prime}}=\frac{2 k^{2} \tau_{1}}{\hbar^{2}}\left\{\frac{1+\left(\omega_{c}^{2}+\omega_{L}^{2}\right) \tau_{1}^{2}}{D_{+} D_{-}} \times\left[\left(\gamma_{2} \cos \theta+(\alpha+\beta) \sin \theta \sin \varphi\right)^{2}+\left(\gamma_{1} \cos \theta-(\alpha-\beta) \sin \theta \cos \varphi\right)^{2}\right]\right. \\
& \left.+\frac{1}{1+\omega_{C}^{2} \tau_{1}^{2}} \times\left[\left(\gamma_{2} \sin \theta-(\alpha+\beta) \cos \theta \sin \varphi\right)^{2}+\left(\gamma_{1} \sin \theta+(\alpha-\beta) \cos \theta \cos \varphi\right)^{2}\right]\right\}
\end{aligned}
$$




$$
\begin{aligned}
\Gamma_{x^{\prime} y^{\prime}}= & \frac{2 k^{2} \tau_{1}}{\hbar^{2}}\left\{\frac{\left[\left(\omega_{c}^{2}-\omega_{L}^{2}\right) \tau_{1}^{2}-1\right] \omega_{L} \tau_{1}}{D_{+} D_{-}} \times\left[\left(\gamma_{2} \cos \theta+(\alpha+\beta) \sin \theta \sin \varphi\right)^{2}+\left(\gamma_{1} \cos \theta-(\alpha-\beta) \sin \theta \cos \varphi\right)^{2}\right]\right. \\
& +\frac{1}{1+\omega_{C}^{2} \tau_{1}^{2}} \times\left[-\left(\alpha^{2}-\beta^{2}\right) \cos \theta \omega_{C} \tau_{1}+\left((\alpha-\beta)^{2}-(\alpha+\beta)^{2}\right) \cos \theta \cos \varphi \sin \varphi\right. \\
& \left.\left.+\gamma_{1}\left[(\alpha-\beta) \sin \theta \sin \varphi-(\alpha+\beta) \omega_{C} \tau_{1} \sin \theta \cos \varphi\right]+\gamma_{2}\left[(\alpha-\beta) \omega_{C} \tau_{1} \sin \theta \sin \varphi+(\alpha+\beta) \sin \theta \cos \varphi\right]\right]\right\}
\end{aligned}
$$

$$
\begin{aligned}
\Gamma_{y^{\prime} x^{\prime}}= & \frac{2 k^{2} \tau_{1}}{\hbar^{2}}\left\{\frac{\left[1-\left(\omega_{c}^{2}-\omega_{L}^{2}\right) \tau_{1}^{2}\right] \omega_{L} \tau_{1}}{D_{+} D_{-}} \times\left[\left(\gamma_{2} \cos \theta+(\alpha+\beta) \sin \theta \sin \varphi\right)^{2}+\left(\gamma_{1} \cos \theta-(\alpha-\beta) \sin \theta \cos \varphi\right)^{2}\right]\right. \\
& +\frac{1}{1+\omega_{C}^{2} \tau_{1}^{2}} \times\left[\left(\alpha^{2}-\beta^{2}\right) \cos \theta \omega_{C} \tau_{1}+\left((\alpha-\beta)^{2}-(\alpha+\beta)^{2}\right) \cos \theta \cos \varphi \sin \varphi\right. \\
& \left.\left.+\gamma_{1}\left[(\alpha-\beta) \sin \theta \sin \varphi+(\alpha+\beta) \omega_{C} \tau_{1} \sin \theta \cos \varphi\right]+\gamma_{2}\left[(\alpha-\beta) \omega_{C} \tau_{1} \sin \theta \sin \varphi+(\alpha+\beta) \sin \theta \cos \varphi\right]\right]\right\}
\end{aligned}
$$

with $k=\sqrt{2 m E_{F}}$ and $D_{ \pm}=1+\left(\omega_{L} \pm \omega_{C}\right)^{2} \tau_{1}^{2}$.

[1] I. Zutic, J. Fabian and S. Das Sarma, Rev. Mod. Phys. 76,323 (2004), and references therein.

[2] S. A. Wolf, D. D. Awswchalom, R. A. Buhrman, J. M. Daughton, S. Von. Molnar, M. L. Roukes, A. Y. Chtchelkanova, D. M. Tresger, Science294, 1488 (2001), and references therein.

[3] S. Datta and B. Das, Appl. Phys. Lett. 56, 665 (1990).

[4] J. Schliemann, J. Carlos Egues and Daniel Loss, Phys. Rev. Lett. 90, 146801 (2003).

[5] E. I. Rashba and Al. L. Efros, Phys. Rev. Lett. 91, 126405 (2003).

[6] L. S. Levitov and E. I. Rashba, Phys. Rev. B 67, 115324 (2003).

[7] S. Murakami, N. Nagaosa and S. C. Zhang, Science 301, 1348 (2003).

[8] M. I. Dyakonov and V. Yu. Kachorovskii, Fiz. Tverd. Tela (leningrad)13,3581 (1971) [Sov. Phys. Solid state 13, 3023 (1971)].

[9] M. I. Dyakonov and V. Yu. Kachorovskii, Fiz. Techn. Poluprov. 20, 178(1986) [Sov. Phys. Semicond. 20,110 (1986)].

[10] E. I. Rashba, Fiz. Tverd. Tela(leningrad) 2, 1224 (1960) [Sov. Phys. Solid state 2, 1109(1960)].

[11] Yu. A. Bychkov and E. I. Rashba, Pis'ma Zh. Eksp. Teor. Fiz. 39, 66(1984) [JETP LETT. 39,78(1984)].
[12] Yu. A. Bychkov and E. I. Rashba, J. Phys. C 17, 6093 (1984).

[13] G. Dresselhaus, Phys. Rev. 100, 580 (1955).

[14] N. S. Averkiev and L. E. Golub, Phys. Rev. B 60, 15582 (1999).

[15] N. S. Averkiev, L. E. Golub and M. Willander, J. Phys. Condens. Matter 14, R271 (2002).

[16] M. M. Glazov, Phys. Rev. B70, 195314 (2004).

[17] B. A. Bernevig, J. Orenstein and S. C. Zhang, cond-mat/0606196 (2006).

[18] P. Q. Jin, Y. Q. Li and F. C. Zhang, J. Phys. A: Math. Gen. 39, 7115 (2006).

[19] G. E. Pikus and A. N. Tikov, in Optical Orientation, Modern Problems in Condensed Matter Science, Vol.8 edited by F. Meier and B. P. Zakharchenya (NorthHolland, Amsterdam), p.109.

[20] M. I. Dyakolov and V. I. Perel, Zh. Eksp. Teor Fiz. 65,362 (1973) [Sov. Phys. JETP 38, 177]

[21] M. M. Glazov and E. L. Ivchenko, J. Supercond. 16, 735 (2003).

[22] E. L. Ivchenko, Fiz. Tverd. Tela (leningrad) 15, 1566 (1973) [Sov. Phys. Solid State 15, 1048 (1973).

[23] M. M. Glazov, private communication. 